\title{
Center for International Blood and Marrow Transplant Research
}

National Cancer Institute

\section{Source}

National Cancer Institute. Center for International Blood and Marrow Transplant

Research. NCI Thesaurus. Code C106396.

A combined research program of the National Marrow Donor Program and the Medical College of Wisconsin that collaborates with the global scientific community to advance hematopoietic cell transplantation and cellular therapy research worldwide. 